\title{
Time Travel: Time Dilation
}

\author{
Daniel Buzzo \\ University of the West of England \\ Department of Computer Science and Creative Technology, \\ Coldharbour Lane, \\ Bristol, UK \\ daniel.buzzo@uwe.ac.uk
}

\begin{abstract}
In this paper I discuss the theory and actuality of the effects of Time Dilation, as predicted by Einstein's Special Relativity. Alongside this the research investigates the visible, experiential effects of most personal form of Time Travel from a personal, phenomenological perspective, illustrated through digital media.
\end{abstract}

The body of work, this paper and an accompanying book of modified digital images, recalls the Hafele-Keating experiment of October 1971 and parallels the Quantified Self experiments of diary photography and personal analytics in a holistic, interconnected way.

The work tracks 12 months of air travel and illustrates with practical data the physics and mathematics behind the theory and actuality of Time Dilation. The research combines the interdisciplinary worlds of Computer Arts with Data and Scientific Visualisation to create a tangible collision between the visualisation of the personal and illustration of theory

The observations are documented in ethnographic, photo essay illustrations as an investigation into the mathematics and physics of personal and interpersonal time. Using situated imaging presented as a practice-based body of work to communicate the abstract world of very small time periods and the direct relevance of Einstein's work on the personal and perceptual world we inhabit. Contrasting Newtonian mechanics and Einsteinian space-time the work seeks to illustrate the personal nature of how the reality of time travel influences every aspect of the interpersonal

Time dilation. Time travel. General relativity. Special relativity. Visual thinking. Photography. Visualisation. Practice-based research. Art.

\section{INTRODUCTION}

In 1971 two American researchers, J.C. Hafele and R. E. Keating, conducted an experiment.

They placed two caesium atomic clocks on passenger airplanes and flew them in opposite directions around the globe to test a hypothesis first posited in Einstein's theory of special relativity.

Their aim was to measure the effects of relativity on these highly accurate devices - the predictions indicated that the eastward flying clock would lose $40 \mathrm{~ns}$ and the westward gain $275 \mathrm{~ns}$. A nanosecond is equal to one billionth of a second $\left(10^{-9}\right.$ or $1 / 1,000,000,000 \mathrm{~s})$. To compare, one nanosecond is to one second as one second is to 31.7 years.

During October, 1971, four cesium atomic beam clocks were flown on regularly scheduled commercial jet flights around the world twice, once eastward and once westward, to test Einstein's theory of relativity with macroscopic clocks. From the actual flight paths of each trip, the theory predicted that the flying clocks, compared with reference clocks at the U.S. Naval Observatory, should have lost $40+/-23$ nanoseconds during the eastward trip and should have gained $275+/-21$ nanoseconds during the westward trip ... Relative to the atomic time scale of the U.S. Naval Observatory, the flying clocks lost $59+/-10$ nanoseconds during the eastward trip and gained $273+/-7$ nanosecond during the westward trip, where the errors are the corresponding standard deviations. These results provide an unambiguous empirical resolution of the famous clock 'paradox' with macroscopic clocks. (Hafele \& Keating 1972)

The results showed that compared to stationary clocks in the laboratory the eastward clock lost $59 \mathrm{~ns}$ and the westward gained $273 \mathrm{~ns}$. Whilst there has been discussion of the accuracy and therefore reliability of the measurements the experiment is commonly held up as one of the first to give practical evidence of the physical effects of time dilation. 


\section{SPECIAL RELATIVITY AND TIME DILATION}

In special relativity Einstein States that the duration of time will pass at different rates when comparing an object at rest versus an object in motion. The greater the velocity of the object in motion, the closer to the speed of light, c, the greater the effect of what is known as 'time dilation'

The effect of this phenomenon is significant at speeds close to the constant of light speed but becomes exponentially less as the object in question approaches rest.

$$
t^{\prime}=\frac{t}{\sqrt{1-\frac{v^{2}}{c^{2}}}}
$$

Figure 1: The mathematical relationship of time, velocity and the speed of light describing Time Dilation

Unless our velocity is a substantial fraction of the constant speed of light the dilation effect $(\mathrm{Y})$ is approximately 1 . This, combined with our extremely limited lifespan is why we don't immediately notice time dilation at ordinary speeds. The fastest speed most humans will achieve, an airplane travelling near the speed of sound, gives a time dilation where $y=1.0000000000005$.

In other words, 1 second for a passenger on the plane would pass as 1.0000000000005 seconds for a stationary observer.

Time effectively passes more slowly for the traveller relative to the observer.

\section{THE REALITY OF THE EFFECT}

Whilst there is discussion of the validity of the results of the 1971 Hafele \& Keating experiment, particularly around the 'corrections' made to their data at publication, their initial observations are clearly aligned with the predicted results to be expected from the mathematics for time dilation.

As Reinhardt et al (2007) note:

Time dilation is one of the most fascinating aspects of special relativity as it abolishes the notion of absolute time

In their 2007 paper they investigate the effects of time dilation with even more accurate atomic clocks, adding more experimental evidence consistent with special relativity. There are also numerous other experiments, such as those into muon decay, that provide additional practical evidence that the effect described is real and measurable.

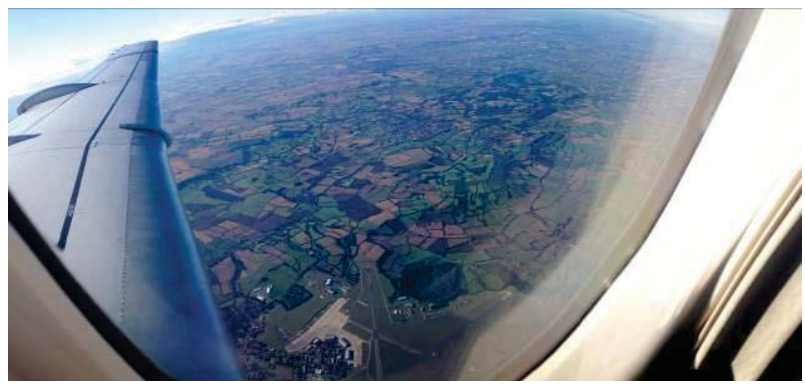

Figure 2: Over Dorset: Duration 55 minutes

\section{IMPLICATIONS FOR EVERYDAY LIFE}

The practical implications for everyday life at first appear intangible when one considers the seemingly miniscule time periods being discussed. Even taking some of the most extreme examples one can imagine being feasible, such as that of a cosmonaut living in a fast low earth orbit, circumnavigating the earth every 90 minutes gives $\mathrm{y}$ of approximately 1.0000000003 .

If one considers our astronaut in orbit for two years, the Time Dilation due to special relativity would give an increase in lifespan of approximately 20 milliseconds. A millisecond is a thousandth $\left(10^{-3}\right.$ or $1 / 1,000$ ) of a second.

This gap of 20 milliseconds, or two hundredths of a second, are suddenly well within human scale perception and understanding. This is a common measured gap in motor racing and fast sports like downhill skiing. Even the record for the $100 \mathrm{~m}$ sprint is now is 9.58 seconds, run by Usain Bolt at the World Athletics championships in Berlin in 2009.

Scales in milliseconds are spaces and durations that even humans with our modest lifespans can perceive and understand.

As Ed Lu, Science Officer on the International Space Station writes, from orbit, in his blog post on experiments in 'Relativity':

After our 6 months in space, we will have actually aged slightly less than everyone else on the ground because of an effect called time dilation. It isn't by much (about 0.007 seconds), but it is one side benefit of flying in space!

Though the effects of time dilation at human scale speeds are often imperceptibly small they are nonetheless real. Seeing the implications of this reflected in everyday life reveals the truly personal nature of the time travel that we undertake in everything we do.

The realisation that time dilation is a tangible and observable phenomena that affects ourselves and everything around us was given heightened 
poignancy by spending large amounts of time flying at altitude and high speed. Observing the curvature of our planet and seeing the dark sky at the edge of space overhead, watching the mountains and deserts as they slid effortlessly under the wings of successive aeroplanes. Being able to observe the size of the individual against the backdrop of a macro-cosmic scale all the while reflecting on the fact that to a subatomic particle travelling near the speed of light, the universe is about four weeks old and it can be crossed in a matter of months.

Nature can produce even larger particle energies. Some particles striking the Earth's upper atmosphere have energies that exceed $2 * 1020 \mathrm{eV}$. If such particles are protons (with mass of about $1 \mathrm{GeV}$ ), their speeds would be 0.999999999999999999999995 c. For them, $\mathrm{y}$ is 1011. Now the age of the universe is about 13 billion years for us, but for such particles, the age of the universe would be about (13 billion years/1011), ie about a month. Such a particle could cross the visible universe in a matter of months (their time).

This twist of perspective, the deliberate 'making strange' that Shlovsky (1917) describes occurs when translating the abstract of the macrocosmic to the intimacy of the personal. The act of comparing and contrasting macro-scale physics and mathematics with recordings of human scale observations evidence reveals useful and potentially significant insights into the nature of the world we actually inhabit.

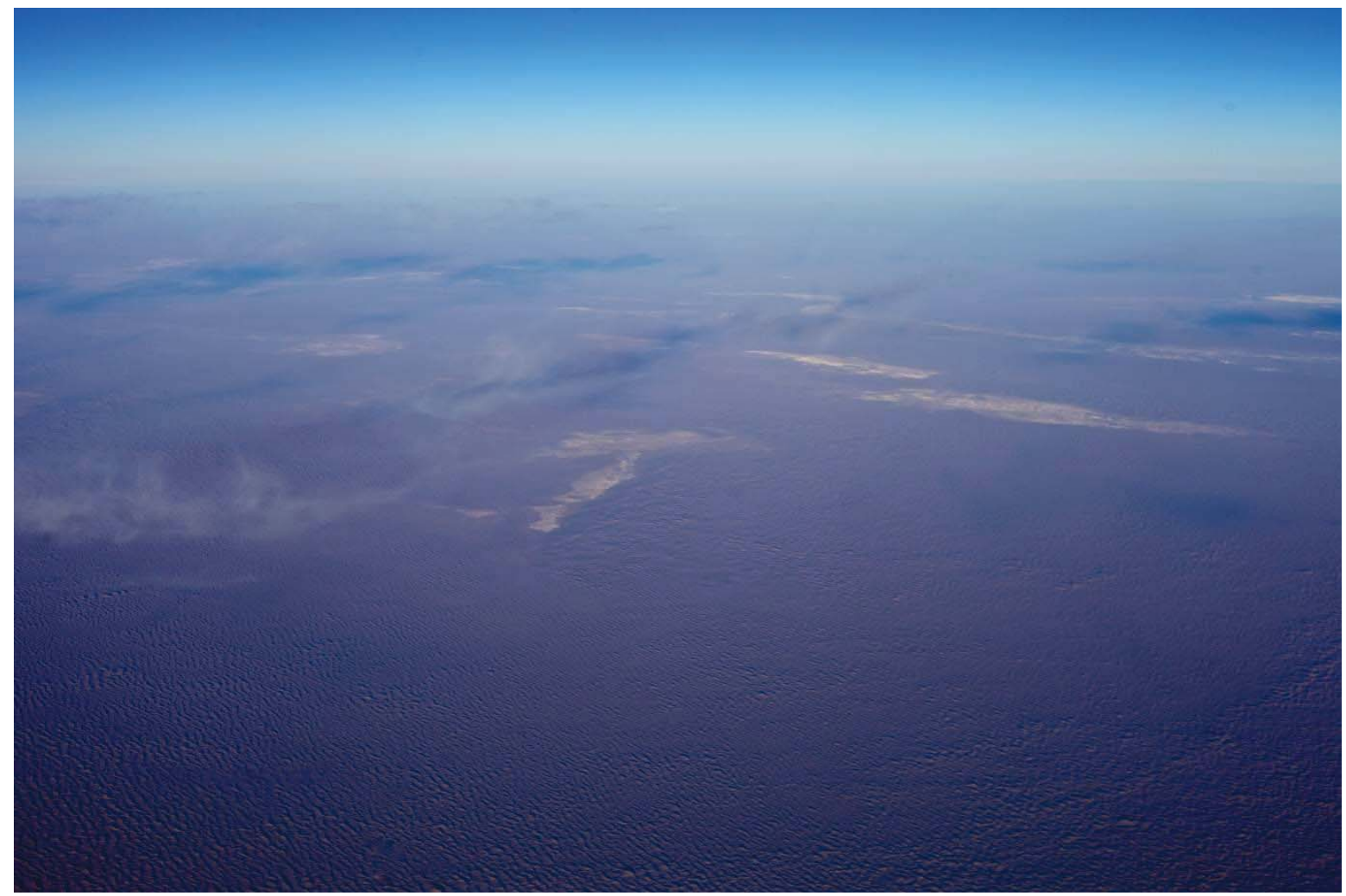

Figure 3: Sunset over Northern China. Duration: 10 hours 50 minutes

\section{THE PICTORIAL AS PRACTICE-BASED RESEARCH}

As Blevis (2011) so eloquently puts it, as an introduction to the extensive list of uses of digital imagery in design thinking.

Visual thinking is the use of imagery and other visual forms to make sense of the world and to create meaningful content. Digital imagery is a special form of visual thinking, one that is particularly salient for $\mathrm{HCl}$ and interaction design
Alongside the descriptions of digital imagery as a 'form of information' and a 'shared and externalised memory and cognition' (Blevis 2012) I would argue that practice-based research, when used as an investigative tool, can assist in revealing and documenting subtle but important non-verbal evidence and also provide compelling and persuasive arguments within presentation of research findings and conclusions. The research approach taken in this area is explicitly practicebased, rooted within contemporary lens based digital media and video art. The investigative material presented as the counterpart to this research paper was collated from hundreds of 
minutes of digital video and several hundreds of digital still images taken while airborne on commercial passenger aircraft during January December 2013. Taken over the course of 44 flights, totalling over 80 hours airborne the visual material is published as a 42 page visual essay, also containing text elements and descriptions taken from this academic paper.

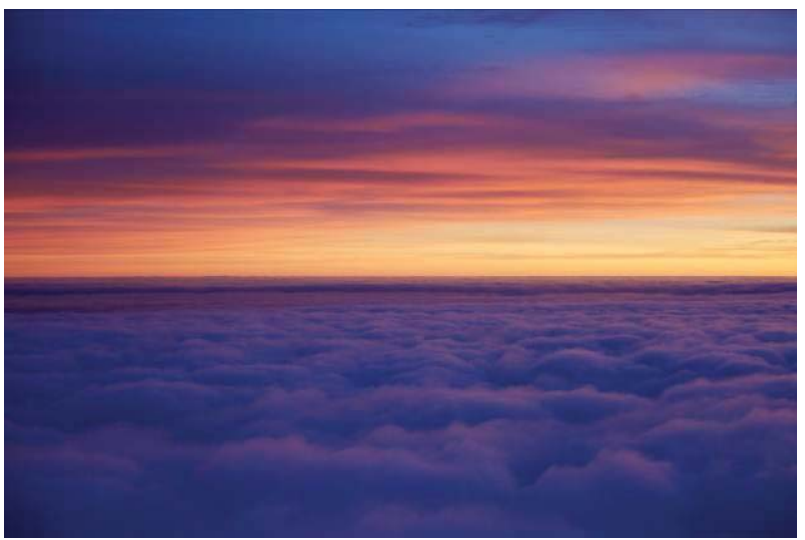

Figure 4: Sunset over London. Duration: 55 minutes

\section{REALISATION OF EFFECT ON THE PERSONAL}

Having recorded the number of flights, durations and distances the calculations based upon the mathematics of special relativity, as discussed earlier show that.

Given 44 flights totalling 85 hours and 20 minutes or approximately 5120 minutes flying time. gives 307,200 seconds of travel per year.

If $y=1.0000000000005$ when travelling at or near the speed of sound, gives a dilation of 0.0000001536 seconds.

To slip one second from base reference, such as the clock sat on my kitchen table I need to travel at my present annual rate for 6,510,416 Years.

Or to put it a different way, travel for a little over 260,000 years to slip one frame out of sync on a standard rate video camera.

\section{CONSIDERING PHOTOGRAPHY IN EVIDENCE}

When collating the increasing amounts of video and photographic material recorded whilst airborne, travelling at or near the speed of sound, the overwhelming sensation of velocity, of speed, of travel is ever-present. Viewed at a macro scale the visual material illustrates this distancing, sliding nature of objects moving in a larger framework. It supports the realisation that the terra firma so often taken as a baseline for observation is in itself only a small part of a larger perspective. The perspective that the proprioceptive nature of our observations of time, informed by the physicality of the form we have evolved and the life span perspective we consider as a norm and useful context are challenged by the linking of these measurable effects. The effects of the micro-scale variations in time seen in contrast to the macro-scale movement around the curve of the earth.

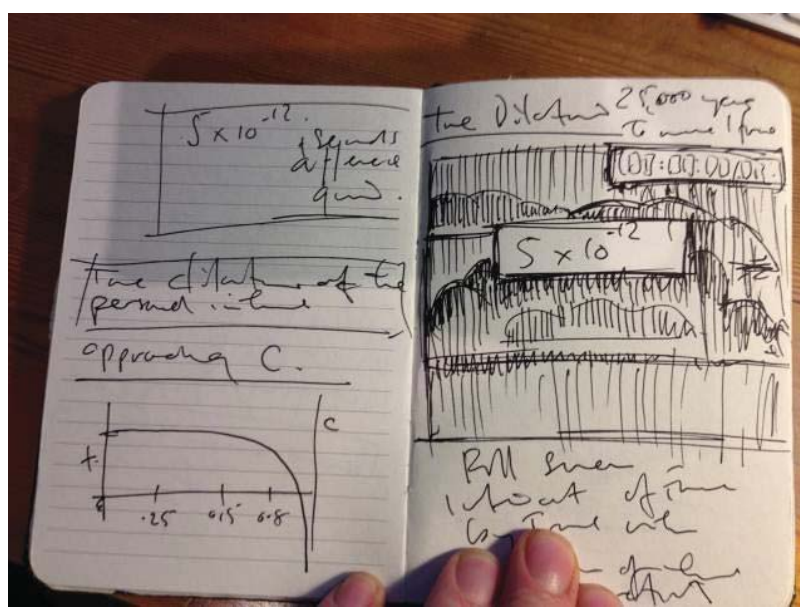

Figure 5: An airplane travelling near the speed of sound gives a time dilation where $y=1.0000000000005$

The visual material, when presented as an adjunct to the evidence of the universal but intimately personal nature of the effects of time dilation help communicate the simple fact that we are all time travelling. Pursuing our own clearly delineated paths and durations relative to each other. Rather than there being one universal time that exists everywhere, for everyone, all at the same moment the personal implication that there is real evidence to prove that this is an illusion is both moving and compelling.

Sitting in an airline seat riding at the edge of space, looking down on the oceans, seas, cities, forests and deserts, seeing terra firma for the illusion that it is. Not a constant underlying grid of space and time - the universal yard stick and the universal clock, such as Newtonian physics promised as it replaced the Aristotelian events-make-time view of the world that had reigned before in popular consciousness.

Realising that the H\&K caesium clock from 1971 could be sat beside me, ticking away in its own little timetrack, ticking to a completely separate rhythm than its counterpart clock sat on my kitchen table at home. Realising that the slipping and sliding of our lives against each other is our own intimate version of time travel, we all move forward as future becomes present becomes past but the durations we experience, and that actually occur, and when now happens is subtly different for everyone. The 
time we each experience actually does expand and contract as we move in space.

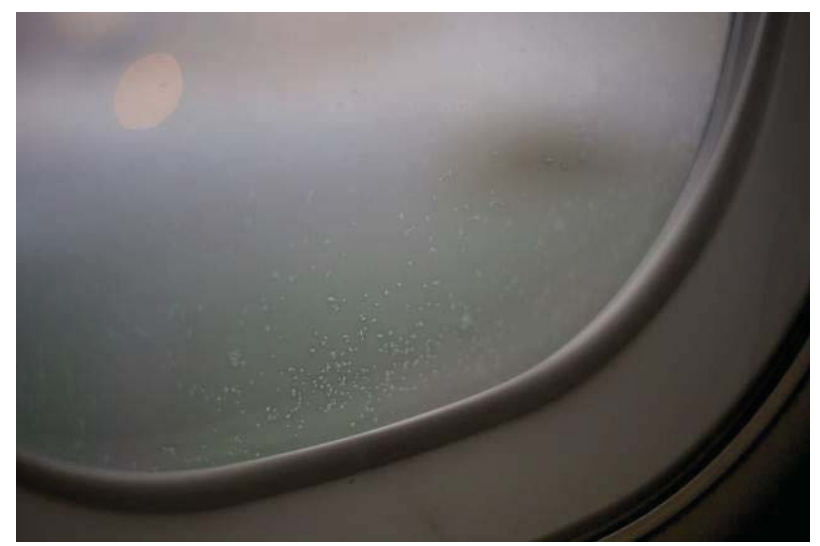

Figure 6: Frost over Mongolia. Duration: 10 hours 55 minutes

The illusion of the Newtonian ideal of a universal timescale, the idea of a 'universal now' is disappearing and being replaced by the ideas of general relativity. Of a universe full of individual 'nows' all unique and all moving independently. This change is akin to seeing the universe of unique, individual 'nows' at a human, personal scale and seeing a life travelling, sliding, back and forth in time against the 'nows' of those around one.

The important element this body of work seeks to describe is not physics or even a discussion of physics but an explanation of physics and the revelatory moment of seeing it in 'real' experienced life. This work seeks to relate that revelatory moment and articulate it with the artefacts, the book and the paper, telling the story of seeing how each and every one of us is travelling in time, independently, uniquely, every day.

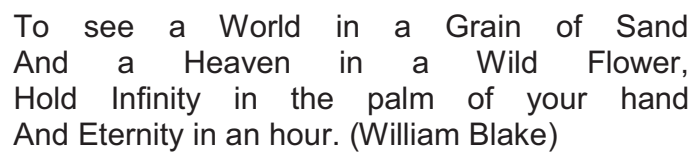

\section{REFERENCES}

Bergson, H. (1923) The Creative Mind: An Introduction to Metaphysics, Citadel Press [1992]

Blevis, E. (2011) Digital imagery as meaning and form in $\mathrm{HCl}$ and design: an introduction to the Visual Thinking Backpage Gallery. Interactions 18, 5 (September 2011), 60-65.

Blevis, E, Churchill. E, Odom. W, Pierce.J, Roedl.D, and Wakkary.R. (2012) Visual thinking \& digital imagery. In Proc. CHI EA '12. ACM, New York, NY, USA, 2715-2718.

Callender, C., and Edney, R. (2001). Introducing Time. Icon Books. ISBN 1-84046-592-1.

Hafele J.C., and Keating. R. E. (1972) Science 177, 166.

Lu. E, 'Expedition 7, Relativity' Blog Post. http://spaceflight.nasa.gov/station/crew/exp7/lulette rs/lu_letter13.html (retrieved 20 March 2014).

Muon Experiment in Relativity. http://hyperphysics.phy-

astr.gsu.edu/hbase/relativ/muon.html (retrieved 5 March 2014).

Reinhardt, S. et al. (2007). Test of relativistic time dilation with fast optical atomic clocks at different velocities. Nature Physics 3 (12): 861-864.

Shklovsky, V. (1917) Art as Technique. In: Russian Formalist Criticism: Four Essays. Trans. Lee T. Lemon and Marion J. Reis. University of Nebraska Press, Lincoln, 1965.

The Quantified Self. http://quantifiedself.com/ (retrieved 4 December 2013).

Toothman, J. How Do Humans age in space? HowStuffWorks.

http://science.howstuffworks.com/humans-age-inspace.htm (retrieved 24 April 2012). 